\title{
Pulmonary Manifestations and Management of COVID-19 Pediatric Patients Admitted in a Tertiary Government Hospital
}

\author{
Wright H. Alborote, MD, Maria Cristina H. Lozada, MD and Kevin L. Bautista, MD \\ Division of Pediatric Pulmonology, Department of Pediatrics, Philippine General Hospital, University of the Philippines Manila
}

\begin{abstract}
Background. Coronavirus Disease 2019 (COVID-19) presents with respiratory signs and symptoms in children. Presently, there are no local studies on the pulmonary manifestations and management of COVID-19 among children.

Objective. Our study aimed to identify and describe the presenting respiratory signs and symptoms, oxygenation status, radiologic findings, blood gas analysis, and pulmonary interventions among children admitted for COVID-19. We also analyzed the clinical and radiologic variables associated with mortality.

Methodology. This is a retrospective study using data obtained from a review of medical records from April 1, 2020, to June 30, 2020, at a tertiary government institution in the Philippines. All pediatric patients (0-18 years) hospitalized for probable or confirmed COVID-19 during the said time period were included in this study. Univariate and multivariate logistic regression was applied to determine factors affecting mortality.
\end{abstract}

Results. A total of 25 pediatric patients with a mean age of 7 years old (age range: 11 days to 18 years) were admitted for COVID-19. Cough (44\%) and dyspnea (24\%) were the most common presenting respiratory symptoms, while tachypnea (68\%), crackles (36\%), and peripheral oxygen desaturation (36\%) were the most common respiratory signs. Indeterminate findings for COVID-19 such as multifocal or diffuse ground-glass opacities and/or consolidations were the most common radiographic abnormalities. Invasive ventilatory support was administered to 6 cases of severe COVID-19 and 4 critical cases. There were no variables that correlated significantly with mortality.

Conclusion. Respiratory signs and symptoms were prominent in our cohort of children admitted due to COVID-19. Mechanical ventilation was required in more severe cases. Larger prospective studies may help identify variables that significantly correlate with poor outcomes among children with COVID-19.

Key Words: COVID-19, children, pulmonary manifestations, management

\section{INTRODUCTION}

Corresponding author: Wright H. Alborote, MD Division of Pediatric Pulmonology Department of Pediatrics Philippine General Hospital University of the Philippines Manila Taft Avenue, Manila 1000, Philippines Email: whalborote@up.edu.ph
The coronavirus disease 2019 (COVID-19) is a viral infection caused by severe acute respiratory syndrome coronavirus 2 (SARS-CoV-2), a member of the $\beta$-coronavirus cluster of enveloped single-stranded RNA viruses. ${ }^{1}$ This novel viral infection initially presented as a series of unexplained pneumonia in Wuhan, China in December 2019, spread rapidly among different regions in the world and was labeled a pandemic by the World Health Organization (WHO). ${ }^{1}$

While the majority of COVID-19 patients develop only mild or moderate disease, approximately $15 \%$ develop a severe disease that requires oxygen support or mechanical ventilation, and 5\% have a critical disease with complications such as respiratory failure, acute respiratory distress syndrome (ARDS), sepsis and septic shock, thrombo- 
embolism, and/or multiorgan failure, including acute kidney injury and cardiac injury. ${ }^{2}$

In the United States, among 149,082 reported COVID cases for which age was specified, 2,572 (1.7\%) were among children aged $<18$ years. ${ }^{3}$ A review of 72,314 cases by the Chinese Center for Disease Control and Prevention, showed that less than $1 \%$ of the cases were in children younger than 10 years old. ${ }^{4}$ In the spectrum of disease severity of COVID-19, most infected children have only mild clinical manifestations, most do not have fever or symptoms of pneumonia. ${ }^{5}$

The main basis for the diagnosis of infection with SARS-CoV-2 is real-time polymerase chain reaction (RTPCR) of respiratory tract secretions. ${ }^{6}$ Co-infection with other infections is reported among pediatrics patients with COVID. ${ }^{7-8}$ Chest $\mathrm{x}$-ray and chest $\mathrm{CT}$ scan may also reveal findings suggestive of COVID-19 in children, such as patchy lesions, ground-glass opacities, consolidation with halo signs, fine mesh shadows and may also be normal. ${ }^{7-9}$

In the Philippines, the first two confirmed COVID-19 cases were previously well adults from China with positive SARS-CoV-2 RT-PCR results in January 2020. ${ }^{10}$ As of September 28, 2020, there are 304,226 cases of COVID-19 nationwide. ${ }^{11}$ Salva et al. reported that among hospitalized patients with suspected COVID-19 in a government hospital in Manila, confirmed cases were more likely to be older, with underlying disease, present with dyspnea, and had a longer symptom duration. ${ }^{12}$

Disease severity ${ }^{2}$ of COVID-19 in children is based on clinical features (Table 1). Furthermore, a patient may be classified as a "confirmed" or "probable" of COVID-1913 based on SARS-CoV-2 RT-PCR results (Table 2).

Presently, however, no published studies are characterizing the pulmonary manifestations and management of COVID-19 among Filipino children. This paper aims to

Table 1. World Health Organization (WHO) classification of disease severity of COVID-19 in children ${ }^{2}$

Disease Severity Clinical Characteristics

Mild Symptomatic patients meeting the case definition for COVID-19 without evidence of viral pneumonia or hypoxia.

Moderate Patients with clinical signs of non-severe pneumonia (cough or dyspnea plus tachypnea based on age-specific cut-off values and/or chest indrawing) but with no signs of severe pneumonia is considered to have a moderate disease. ${ }^{2}$

Severe disease A child with clinical signs of pneumonia plus at least one of the following: central cyanosis or $\mathrm{SpO}_{2}<90 \%$; severe respiratory distress; general danger sign: inability feed, lethargy or unconsciousness, or convulsions or an adolescent with pneumonia symptoms and respiratory rate $>30$ breaths/min; and/or severe respiratory distress; and/or desaturation with $\mathrm{SpO}_{2}<90 \%$ on room air is considered to have severe disease.

Critical disease

Defined by the presence of Acute Respiratory Disease Syndrome (ARDS), sepsis, or septic shock identify and describe the clinical presentation, particularly the presenting respiratory symptoms, oxygenation status, radiologic findings, blood gas analysis, and pulmonary interventions among children admitted for COVID-19. We also aim to determine which clinical and radiologic variables correlate with favorable or unfavorable clinical outcomes (survival or mortality).

\section{METHODOLOGY}

This is a retrospective cohort study conducted at the University of the Philippines - Philippine General Hospital. The primary investigator reviewed the three months data (April 1, 2020, to June 30, 2020) from the Medical Records Section on the said institution.

All pediatric patients (0-18 years) diagnosed and admitted with probable and confirmed COVID-19 aged 0 mo-18 years old admitted from April 1, 2020, to June 30, 2020, at the Philippine General Hospital. Patients classified as COVID-19 suspects upon admission with a negative RTPCR result and with non-COVID-19 presentations were excluded in our study.

The principal investigator reviewed and recorded the data in a coded data collection form. Data collected were anonymized and patient information was kept confidential. All identifying patient data were securely stored in a separate database with corresponding patient codes to protect patient confidentiality. No data will be used for any purpose other than what is intended for this study. Only the data required by the Data Collection Sheet were gathered.

Descriptive statistics such as mean and standard deviation were used to present continuous variables while frequency and percentage for categorical data. Kruskal Wallis was used to compare the variables (continuous), while the Fisher exact test was used for categorical data. One Way Analysis of Variance (ANOVA) was used to compare the variables according to the severity of the disease. Univariate and multivariate logistic regression was applied to determine factors affecting mortality. The level of significance will be 5\%. Medical Statistical software version 19.2.1 was used to carry out statistical calculations.

Table 2. Department of Health (DOH) Case Classification for COVID-1913

\section{Case Classification Definition}

Probable case Suspect case whose testing for COVID-19 is inconclusive or tested positive for COVID-19 but whose test was not conducted in a national or subnational reference laboratory or officially accredited laboratory for COVID-19 confirmatory testing or who died without undergoing confirmatory testing.

Confirmed case Patient who positive for COVID-19 based on laboratory confirmation at the national or subnational reference laboratory or a DOH-certified laboratory testing facility. 
Table 3. Demographic and Clinical Profile of Pediatric Patients with Probable and Confirmed COVID-19

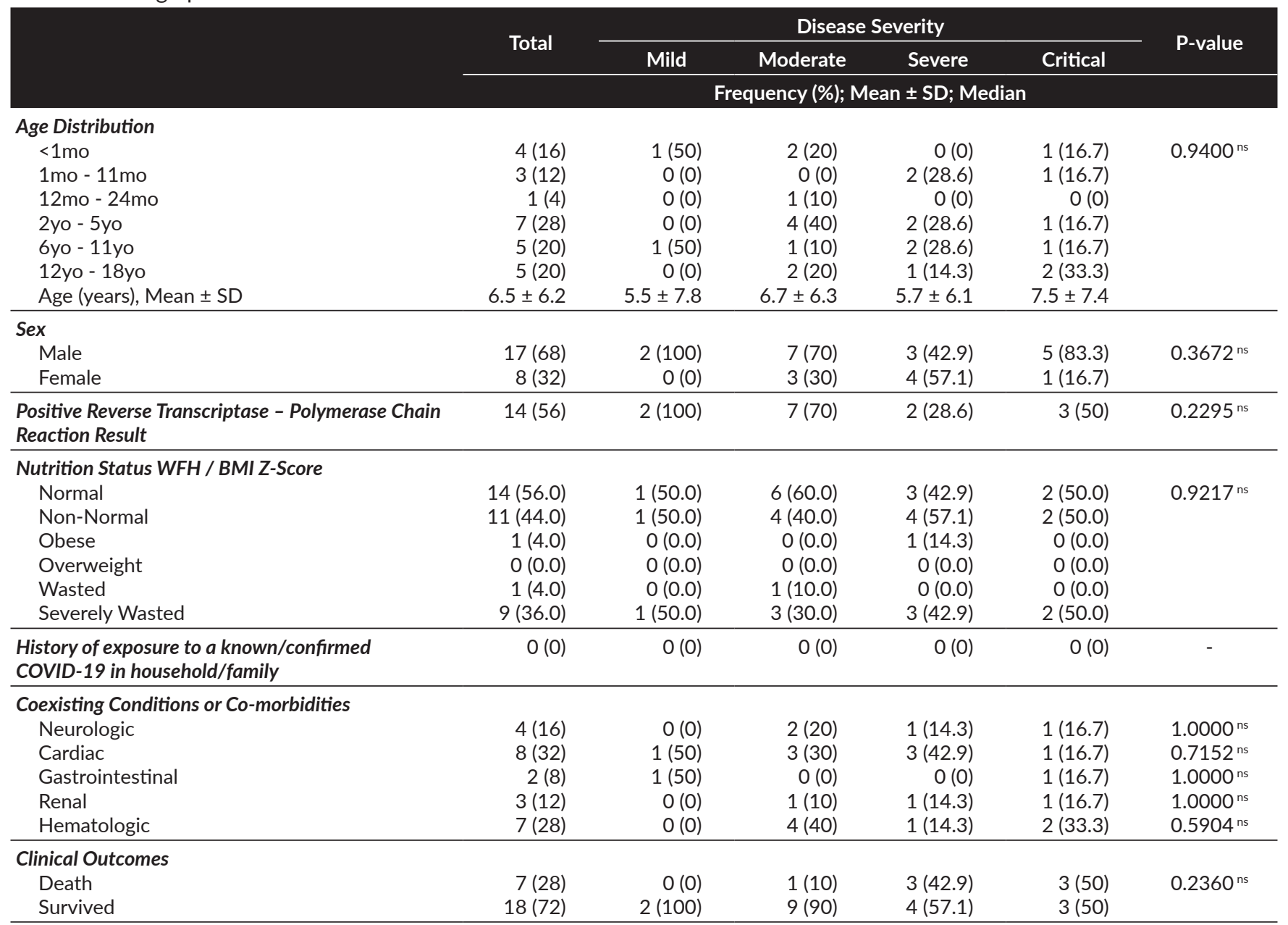

*significant, ns - not significant

The study protocol was submitted to the University of the Philippines Manila Research Ethics Board (UPMREB) for ethics review and approval before implementation. This study commenced only upon approval by the UPMREB (UPMREB Code 2020-464-01).

\section{RESULTS}

\section{Demographic Profile}

There were a total of 25 pediatric patients admitted for probable and confirmed COVID-19 from April 12020 to June 30, 2020, with a mean age of 7 years old (age range of 11 days to 18 years old). Thirty-six percent $(36 \%)$ of patients were severely wasted based on WHO z-scores. All 25 patients had co-existing medical conditions with $32 \%$ having cardiac-related conditions, while $28 \%$ had hematologiconcologic co-morbidities. There were 7 mortalities out of the 25 admitted patients.

Results showed that there was no significant differences between the disease severity groups on their age $(\mathrm{p}=.9400)$, gender $(\mathrm{p}=.3672)$, positive SARS-CoV-2 reverse transcriptase results $(\mathrm{p}=.2295)$, nutrition status or BMI Score $(\mathrm{p}=.751)$, comorbid conditions, and clinical outcome $(\mathrm{p}=.2360)($ Table 3$)$.

Fourteen patients (56\%) were classified as COVID-19 Confirmed Cases while the eleven patients (44\%) were classified as COVID-19 Probable Cases (Table 4).

\section{Presenting Respiratory Signs and Symptoms}

Table 5 shows the clinical manifestations of pediatric patients with probable and confirmed COVID-19. From the data gathered, cough (44\%) and dyspnea (24\%) were the most common initial respiratory symptoms of pediatric patients with probable and confirmed COVID-19. The most common non-respiratory symptoms were fever $\left(38.1^{\circ} \mathrm{C}\right.$ to $\left.39.0^{\circ} \mathrm{C}\right)$, vomiting, and diarrhea. Upon admission to the hospital, tachypnea (68\%), crackles (36\%), and peripheral oxygen desaturation (36\%) were the most common respiratory signs.

The presence of crackles on auscultation was significantly prevalent among patients who were classified as severe (57\%) and critical (67\%) disease. Tachypnea was also 
Table 4. Clinical Features of Confirmed versus Probable COVID-19 cases

\begin{tabular}{lcc} 
& $\begin{array}{c}\text { Confirmed } \\
\text { COVID-19 }(\mathrm{n}=14)\end{array}$ & $\begin{array}{c}\text { Probable } \\
\text { COVID-19 }(\mathrm{n}=11)\end{array}$ \\
\hline Mean Age, range (years) & $5.23(0.03-15)$ & $8.14(0.04-18)$ \\
\hline Sex (Males) & $9 / 14$ & $8 / 11$ \\
\hline Disease Severity & & \\
$\quad$ Mild & $2 / 14$ & $0 / 11$ \\
Moderate & $7 / 14$ & $3 / 11$ \\
Severe & $2 / 14$ & $5 / 11$ \\
Critical & $3 / 14$ & $3 / 11$ \\
\hline Clinical Outcomes & & \\
$\quad$ Death & $1 / 14$ & $6 / 11$ \\
Survived & $13 / 14$ & $5 / 11$ \\
\hline
\end{tabular}

Table 5. Clinical Manifestations of Pediatric Patients with Probable and Confirmed COVID-19

\section{$\mathrm{n},(\%)$}

\begin{tabular}{lc}
\hline Presenting Symptoms & \\
Respiratory & $14(56)$ \\
Cough & $11(44)$ \\
Dyspnea & $6(24)$ \\
Rhinorrhea & $1(4)$ \\
Non-respiratory & $14(56)$ \\
Fever $\leq 38^{\circ} \mathrm{C}$ & $2(8)$ \\
Fever $38.1-39.0^{\circ} \mathrm{C}$ & $10(40)$ \\
Vomiting & $7(28)$ \\
Diarrhea & $5(20)$ \\
\hline Presenting Signs & \\
Respiratory & $22(88)$ \\
Crackles / Rales & $9(36)$ \\
Decreased breath sounds & $2(8)$ \\
Nasal Flaring & $3(12)$ \\
Oxygen desaturation & $9(36)$ \\
Retractions & $10(40)$ \\
Tachypnea & $17(68)$ \\
Wheezing & $1(4)$ \\
Non-respiratory & $19(76)$ \\
Cardiac Murmur & $5(20)$ \\
Cyanosis & $6(24)$ \\
Delayed Capillary Refill & $4(16)$ \\
Disturbance of consciousness & $9(36)$ \\
Hypotension & $4(16)$ \\
Poor pulses & $6(24)$ \\
Tachycardia & $13(52)$ \\
\hline &
\end{tabular}

observed in patients classified as severe and critical COVID disease. On the other hand, among critical COVID patients, disturbances in consciousness, poor pulses, hypotension, and delayed capillary refill were observed to be more prevalent.

\section{Chest Imaging Findings and Blood Gas Analyses Results}

The most common chest $\mathrm{x}$-ray finding were indeterminate findings for COVID-19, particularly multifocal or diffuse ground-glass opacities and/or consolidations (40\%). The remaining $32 \%$ were atypical findings such as central unilateral or bilateral ground-glass opacities. On the other hand, of the 19 patients who underwent a high-resolution chest $\mathrm{CT}$ scan, the majority have indeterminate findings
Table 6. Radiologic Findings and Blood Gas Analysis of Pediatric Patients with Probable and Confirmed COVID-19

Number of patients (\%)

Chest Radiograph

\section{Indeterminate}

Multifocal or diffuse ground-glass opacities and/or

$10(40.0)$ consolidations

\section{Atypical}

Unilateral segmental or lobar consolidation

Central unilateral or bilateral ground-glass opacities

8 (32.0) and/or consolidation

Pleural effusion

Negative

2 (8.0)

\section{Chest CT scan}

\section{Typical}

Bilateral, peripheral, and/or subpleural ground-glass

$6(24.0)$ opacities and/or consolidation in lower lobe predominant pattern

\begin{tabular}{ll}
\hline $\begin{array}{l}\text { Indeterminate } \\
\text { Unilateral peripheral or peripheral and central } \\
\text { ground-glass opacities and/or consolidation }\end{array}$ & $5(20.0)$ \\
$\begin{array}{l}\text { Multifocal or diffuse ground-glass opacities and/or } \\
\text { consolidation without specific distribution }\end{array}$ & $7(28.0)$ \\
Crazy paving sign & $3(12.0)$ \\
\hline Atypical & \\
Unilateral segmental or lobar consolidation & $1(4.0)$ \\
$\begin{array}{l}\text { Central unilateral or bilateral ground-glass opacities } \\
\text { and/or consolidation }\end{array}$ & $2(8.0)$ \\
Pleural Effusion & $2(8.0)$ \\
CT Scan not done & $6(24.0)$ \\
\hline Arterial Blood Gas Results & \\
\hline$\quad$ Normal acid-base balance & $7(31.8)$ \\
$\quad$ Respiratory acidosis & $3(13.6)$ \\
$\quad$ Respiratory alkalosis & $3(13.6)$ \\
Metabolic acidosis & $8(36.3)$ \\
$\quad$ Metabolic alkalosis & $1(4.5)$ \\
\hline Oxygenation & \\
\hline No hypoxemia & $2(8.0)$ \\
Corrected hypoxemia & $4(16.0)$ \\
Uncorrected hypoxemia & $8(32.0)$ \\
Over-corrected Hypoxemia & $8(32.0)$ \\
\hline ABG not done & $3(12.0)$ \\
\hline
\end{tabular}

of multifocal or diffuse ground-glass opacities and/or consolidations with only 6 (24\%) of the patients showed typical findings of COVID-19.

Arterial blood gas (ABG) taken during admission showed that most patients have an underlying metabolic acidosis (36\%) while $31.8 \%$ showed a normal acid-base balance (Table 6).

\section{Ancillary laboratory results}

Among the infection parameters, leukocytosis was identified in $71 \%$ of cases, while $70 \%$ had elevated C-reactive Protein (CRP) on admission. Procalcitonin levels were elevated level in thirteen out of 25 cases, while among the patients with erythrocyte sedimentation rate (ESR) determination, the latter was elevated in $70 \%$ of cases. 
Table 7. Respiratory and Non-respiratory management of Pediatric Patients with Probable and Confirmed COVID-19

\begin{tabular}{lc}
\multicolumn{1}{c}{ Type of Interventions } & $\mathbf{n}(\%)$ \\
\hline Respiratory Interventions & $6(24)$ \\
Nasal Cannula (1-4lpm) & $6(24)$ \\
Oxygen Facemask (6-10lpm) & \\
\hline Mechanical Ventilation & \\
Invasive & $9(36)$ \\
Non-invasive & $0(0)$ \\
No oxygen support & $3(12)$ \\
Prone Positioning & $1(4)$ \\
\hline Non-respiratory & \\
Intravenous Antibiotics & $23(92)$ \\
Systemic Glucocorticoids & $9(36)$ \\
Immune globulin & $5(20)$ \\
Convalescent Plasma Transfusion & $4(16)$ \\
Transfusion (Blood Products) & $8(32)$ \\
Inotropes & $10(40)$ \\
Vitamin D & $10(40)$ \\
Zinc Sulfate & $11(44)$ \\
Tocilizumab & $3(12)$ \\
Hydroxycholoroquine & $1(4)$ \\
Anti-viral (Lopinavir + Rotinavir) & $2(8)$ \\
\hline
\end{tabular}

\section{Respiratory and Non-Respiratory Management}

Results showed that oxygen therapy via nasal cannula was given to patients classified with moderate severity (60\%). Invasive mechanical ventilation commencing with a $100 \%$ fraction of inspired oxygen $\left(\mathrm{FiO}_{2}\right)$ was given to severe (85.7\%) or critical (66.7\%) cases of pediatric COVID-19.

Supportive treatment such as Vitamin D and Zinc Sulfate was given to $40 \%$ and $44 \%$ of the cases respectively. Meanwhile, inotropic support and medications were given to severe and critical cases. Table 7 lists the respiratory and non-respiratory interventions administered among our cohort of children with probable and confirmed COVID-19.

\section{Factors correlated with mortality}

Table 8 shows the risk factors for mortality based on the clinical and radiologic variables. The presence of crackles was not a significant factor of mortality $(\mathrm{p}=.6559)$ although, among those who died, $42.9 \%$ were observed to manifest with this finding. Furthermore, dyspnea $(\mathrm{p}=0.1826)$ and peripheral oxygen desaturation $(\mathrm{p}=1826)$ had a higher percentage among patients who died but these, too, did not reach statistical significance.

Among the clinical variables, there were no variables that correlated significantly with mortality.

\section{DISCUSSION}

On March 30, 2020, the Philippine General Hospital has been designated as a COVID-19 referral hospital in the Philippines. ${ }^{14}$ We reported data on the pulmonary manifestations and management of pediatric COVID disease and analyzed which parameters correlate with favorable or unfavorable clinical outcomes using a review of medical records of children admitted during the early months of the pandemic.

Pediatric patients with COVID-19 present with distinct epidemiological, clinical, and radiological characteristics., ${ }^{95-16}$ In our study, cough, fever, and dyspnea were the most predominant symptoms from both probable and confirmed COVID-19 patients. Similar results were observed in the study by Zacariah et al. ${ }^{15}$ and $\mathrm{Wu}$ et al. ${ }^{16}$

In our study, the presence of crackles and tachypnea were observed to be significantly higher in severe and critical cases in this study. Oxygen desaturation and crackles were also predominant in severe and critical cases, though not statistically significant. In an article by Shen et al., these signs were included in the criteria for severe and critical cases of COVID $-19 .{ }^{5}$

Table 8. Crude and Adjusted Odds Ratio of Variables which may correlate with mortality among pediatric patients with Probable and Confirmed COVID-19

\begin{tabular}{|c|c|c|c|c|c|c|}
\hline \multirow{2}{*}{ Clinical and Radiologic Variables } & \multicolumn{3}{|c|}{ Crude Odds Ratio } & \multicolumn{3}{|c|}{ Adjusted Odds Ratio } \\
\hline & OR & $95 \%$ & p-value & OR & $95 \%$ & p-value \\
\hline Age less than 2 years old & 0.8 & 0.1 to 5.4 & $0.8189^{\text {ns }}$ & 0.79 & 0.1 to 6.5 & $0.8300^{\text {ns }}$ \\
\hline Male sex & 0.51 & 0.1 to 3.2 & $0.4715^{\mathrm{ns}}$ & 0.43 & 0.1 to 3.3 & $0.4128^{n s}$ \\
\hline Non-Normal Nutritional status & 0.13 & 0.0 to 1.3 & 0.0876 & 0.1 & 0.0 to 1.3 & 0.0787 \\
\hline History of Cough & 0.4 & 0.1 to 2.6 & $0.3400^{\mathrm{ns}}$ & 0.2 & 0.0 to 1.9 & $0.1601^{\mathrm{ns}}$ \\
\hline Presence of Crackles / Rales & 1.5 & 0.3 to 9.0 & $0.6569^{n s}$ & 0.42 & 0.0 to 4.3 & $0.4629 \mathrm{~ns}$ \\
\hline Delayed Capillary Refill & 12.75 & 1.0 to 157.2 & $0.0470^{*}$ & 5.73 & 0.3 to 93.9 & $0.2216^{\mathrm{ns}}$ \\
\hline Disturbance of consciousness & 3.47 & 0.6 to 21.4 & $0.1801^{\mathrm{ns}}$ & 0.64 & 0.0 to 11.7 & $0.7641^{\mathrm{ns}}$ \\
\hline History of Dyspnea & 3.75 & 0.5 to 26.2 & $0.1826^{\mathrm{ns}}$ & 2.38 & 0.3 to 20.4 & $0.4272^{\mathrm{ns}}$ \\
\hline Presence of Fever $38.1-39.0^{\circ} \mathrm{C}$ & 1.18 & 0.2 to 6.90 & $0.8558^{\text {ns }}$ & 0.68 & 0.1 to 5.5 & $0.7132^{\mathrm{ns}}$ \\
\hline Hypotension & 3.2 & 0.4 to 28.9 & $0.3006^{\text {ns }}$ & 0.93 & 0.1 to 12.6 & $0.9565^{\mathrm{ns}}$ \\
\hline Oxygen Desaturation & 3.47 & 0.6 to 21.4 & $0.1801^{\mathrm{ns}}$ & 1.37 & 0.1 to 12.7 & $0.7814^{\mathrm{ns}}$ \\
\hline Poor pulses & 10.67 & 1.3 to 86.9 & $0.0270^{*}$ & 5.31 & 0.4 to 66.7 & $0.1960^{\mathrm{ns}}$ \\
\hline Retractions & 1.18 & 0.2 to 6.9 & $0.8558^{\text {ns }}$ & 0.7 & 0.1 to 5.2 & $0.7248^{n s}$ \\
\hline Tachycardia & 0.6 & 0.1 to 3.5 & 0.5699 ns & 0.3 & 0.0 to 2.6 & $0.2787^{\mathrm{ns}}$ \\
\hline Tachypnea & 1.25 & 0.2 to 8.4 & $0.8189^{n s}$ & 0.37 & 0.0 to 5.0 & $0.4548^{n s}$ \\
\hline Atypical finding on chest radiograph & 0.7 & 0.1 to 4.5 & $0.7084^{\text {ns }}$ & 0.99 & 0.1 to 7.9 & $0.9890^{\mathrm{ns}}$ \\
\hline
\end{tabular}

*significant, ns - not significant 
Our study also showed that none of the pulmonary manifestations mentioned increases the risk of mortality Similarly, on review of adult studies on the predictors of mortality in patients with COVID-19, no clinical manifestations were included as risk factors for mortality. ${ }^{17,18}$

We observed that the most predominant finding on chest $\mathrm{x}$-ray was an indeterminate finding of COVID-19 pneumonia with multifocal or diffuse ground-glass opacities and/or consolidations (40\%). In our institution, the reporting of chest $\mathrm{x}$-ray and chest $\mathrm{CT}$ findings (i.e., typical, atypical, indeterminate) were by the recommended chest imaging in the pediatric COVID-19 consensus statement by Foust and colleagues. ${ }^{1}$

Based on the paper by Foust et al., chest $x$-rays in pediatric COVID-19 pneumonia may show patchy opacities with peripheral and lower lung zone predominance ${ }^{1}$, however, may be less sensitive than CT Scan for detecting parenchymal abnormalities. ${ }^{1}$ In a large systematic review conducted by Hoang et al., patchy lesions, and ground-glass opacities were commonly reported in the chest radiograph. ${ }^{19}$ Normal chest $\mathrm{x}$-ray, pleural effusion, and altered cardio-mediastinal contour were the uncommon findings radiograph findings in a study by Serrano et al., which is also similar to our results. ${ }^{20}$

In pediatric patients with COVID-19, the common findings on chest CT scan were bilateral peripheral and/or subpleural ground-glass and/or consolidative opacities often in the lower lobes of the lungs and the presence of a halo sign. ${ }^{1,7}$ Of the 19 patients who underwent chest CT scan in our study, only 6 patients (24\%) presented with the said findings. No halo signs were noted on the chest CT scan. The majority showed indeterminate finding of COVID-19 pneumonia. In our study, both chest $\mathrm{x}$-ray and chest $\mathrm{CT}$ scan results were not significant factors for mortality.

Nucleic acid testing by reverse transcriptase-polymerase chain reaction (RT-PCR) is regarded as the main method for laboratory confirmation of COVID-19 disease..$^{21}$ Other ancillary tests may have variable findings. The white blood cell count is usually normal or reduced, while the $\mathrm{C}$-reactive protein (CRP) may be normal or elevated..$^{21}$ Procalcitonin levels are normal in most cases, while levels of $>0.5 \mathrm{ng} / \mathrm{mL}$ may suggest bacterial co-infection. ${ }^{21}$ In our cohort, most of the patients showed elevated infection parameters, but none were identified to correlate significantly with mortality.

Based on the pediatric COVID-19 management protocol by Carlotti et al., the four main principles for adequate therapeutic management are early identification, isolation, diagnosis, and treatment. ${ }^{22}$ Severe cases with respiratory distress and/or hypoxia warrant specialized care and should be admitted, specifically, those with respiratory failure requiring mechanical ventilation, shock, and organ dysfunction. ${ }^{22}$

In our study, most of the patients belong to moderate, severe, and critical severity. Mechanical ventilation was the primary respiratory management administered in patients who presented with severe and critical signs on admission.
Oxygen therapy through nasal cannula and facemask were given to less severe cases. Invasive ventilatory support was administered to 6 cases of severe COVID and 4 critical cases.

The optimal mode of respiratory support for pediatric patients with COVID-19 presenting with hypoxemia remains to be explored. Present guidelines suggest the use of a high-flow nasal cannula or non-invasive modes of ventilation such as non-invasive positive pressure ventilation or bubble continuous positive airway pressure before invasive mechanical ventilation. ${ }^{2,23}$ Protective mechanical ventilation strategies using lower tidal volumes (around 4-6 mL/kg), target plateau pressure of 28 to $30 \mathrm{cmH}_{2} \mathrm{O}$, and positive endexpiratory pressure (PEEP) titration on a case-to-case basis is advised $^{22-23}$ Prone positioning may also be helpful, especially for patients with $\mathrm{PaO}_{2} / \mathrm{FiO}_{2}$ of $<150 \mathrm{mmHg}$. 22 In our study, there were no patients who underwent a trial of highflow nasal cannula, as this mode of respiratory support for pediatric patients is not yet available in our institution at the time of this writing. Only one patient with severe COVID was observed to be placed on prone.

\section{Limitations of the Study}

The findings of our study may be limited by its retrospective nature and the small sample size which may have precluded identification of other clinical and radiologic variables that significantly correlate with mortality. Another limitation is that since our institution was designated as a COVID referral center to serve patients with more severe illnesses, data on mild or asymptomatic cases were very limited. Furthermore, our findings may not be representative of data from other geographic areas in the Philippines.

\section{Recommendations for Further Study}

In line with said limitations, we recommend a prospective study with a larger sample size and additional clinical, radiologic, and laboratory variables. Multi-center studies on the respiratory manifestations and management of pediatric COVID may be recommended for further study.

\section{CONCLUSION}

This study showed that the most common pulmonary manifestations of probable and confirmed COVID-19 among children who were admitted to our institution were cough, dyspnea, tachypnea, retractions, and crackles. Other common non-pulmonary manifestations include fever, tachycardia, and disturbances of consciousness. There were no clinical and radiologic variables significantly associated with mortality; however, the small sample size may have precluded the determination of variables with statistical significance. Respiratory management primarily given to patients classified as severe and critical upon admission was invasive mechanical ventilation while oxygen therapy through nasal cannula and oxygen facemask were given to less severe cases. 


\section{Statement of Authorship}

All authors participated in data collection and analysis, and approved the final version submitted.

\section{Authors Disclosure}

All authors declared no conflicts of interest.

\section{Funding Source}

This paper was self-funded.

\section{REFERENCES}

1. Foust AM, Phillips GS, Chu WC, Daltro P, Das KM, Garcia-Pena $\mathrm{P}$, et al. International expert consensus statement on chest imaging in pediatric COVID-19 patient management: imaging findings, imaging study reporting and imaging study recommendations. Radiology: Cardiothoracic Imaging. $2020 \mathrm{Apr} 23 ; 2(2)$.

2. World Health Organization. Clinical Management of COVID-19 Interim Guidance. World Health Organization May 27, 2020. [Internet] Available from: https://www.who.int/publications/i/item/ clinical-management-of-covid-19.

3. Centers for Disease Control and Prevention COVID-19 Response Team. Coronavirus disease 2019 in children - United States, February 12-April 2, 2020. MMWR Morb Mortal Wkly Rep. 2020 Apr 10. 69 (14):422-6.

4. Lu X, Zhang L, Du H, Zhang J, Li Y, Qu J, et al. SARS-CoV-2 infection in children. N Engl J Med 2020; 382(17):1663-5.

5. Shen K, Yang Y, Wang T, Zhao D, Jiang Y, Jin R, et al. Diagnosis, treatment, and prevention of 2019 novel coronavirus infection in children: experts' consensus statement. World J Pediatr 2020 Feb; (7): 1-9

6. Zimmerman P, Curtis N. Coronavirus Infections in Children Including COVID-19: An Overview of the Epidemiology, Clinical Features, Diagnosis, Treatment and Prevention Options in Children. The Pediatric Infectious Disease Journal: May 2020; 39(5):355-68.

7. Xia W, Shao J, Guo Y, Peng X, Li Z, Hu D. Clinical and CT features in pediatric patients with COVID-9 infection: Different points from adults. Pediatric Pulmonology. 2020;55:1169-74.

8. Dosanjh A. Pediatric Coronavirus Disease 2019 and Radiographic Screening:

9. Identification of Patients at Risk. Pediatric Allergy, Immunology, and Pulmonology Jun 2020; 53-6.

10. Ma H, Hu J, Tian J, Zhou X, Li H, Laws MT, Wesemann LD, et al. A single-center, retrospective study of COVID-19 features in children: a descriptive investigation. BMC Medicine 2020; 18:123.
11. Edrada EM, Lopez EB, Villarama JB, Villarama EP, Dagoc BF, Smith C, Sayo AR. First COVID-19 infections in the Philippines: a case report. Trop Med Health. 2020 Apr 14;48:21

12. Department of Health. COVID-19 Tracker Philippines. [Internet] Available from https://www.doh.gov.ph/covid19tracker.

13. Salva EP, Villarama JB, Lopez EB, Sayo AR, Villanueva AM, Edwards $\mathrm{T}$, et al. Epidemiological and clinical characteristics of patients with suspected COVID-19 admitted in Metro Manila, Philippines. Trop Med Health. 2020; 48: 51.

14. Department of Health. Department Memorandum No. 2020-0245. Advisory: Endorsement of the DILG Memorandum "Updated Contact Tracing Report Template for the Coronavirus Disease2019 (COVID-19) Situation" dated May 26,2020. [Internet] Available from: https://www.doh.gov.ph/sites/default/files/health-update/ dm2020-0245.pdf

15. Llaneta CA. UP-PGH begins operating as COVID-19 referral center/ [Internet] Available from https://www.up.edu.ph/up-pgh-beginsoperating-as-covid-19-referral-center.

16. Zachariah P, Johnson, C, Halabi KC, Ahn D, Sen AI, Fischer A. Epidemiology, Clinical Features, and Disease Severity in Patients With Coronavirus Disease 2019 (COVID-19) in a Children's Hospital in New York City, New York. JAMA Pediatr. 2020;174(10):e202430.

17. Qin W, Xing Y, Shi L, Li W, Gao Y, Pan S. et al., Coinfection and Other Clinical Characteristics of COVID-19 in Children. Pediatrics 2020 July; 146 (1): e20200961.

18. Tian W, Jiang W, Yao J, Nicholson CJ, Li RH, Sigurslid HH, et al. Predictors of mortality in hospitalized COVID-19 patients: A systematic review and meta-analysis. J Med Virol 2020 May 22;10.1002/jmv.26050.

19. Aly MH, Rahman SS, Ahmed WA, Alghamedi MH, Shehri AA. Indicators of Critical Illness and Predictors of Mortality in COVID-19 Patients. Infect Drug Resist 2020 Jun 26;13:1995-2000.

20. Hoang A, Chorath K, Moreira A, Evans M, Morton F, et al. COVID-19 in 7780 pediatric patients: A systematic review. EClinicalMedicine 2020 Jun 26;24:100433.

21. Serrano CO, Alonso E, Andres M, Buitrago NM, Vigara AP, Pajares MP, López E, et al , Pediatric chest x-ray in covid-19 infection. Eur J Radiol 2020 Oct;131:109236.

22. Chen Z, Fu JF, Shu Q, Chen YH, Hua C, Li F, et al., Diagnosis and Treatment Recommendations for Pediatric Respiratory Infection Caused by the 2019 Novel Coronavirus. World J Pediatr 2020 Jun;16(3):240-246.

23. Carlotti AP, Carvalko WB, Johnston C, Rodriguez IS, Delgado AF. COVID-19 Diagnostic and Management Protocol for Pediatric Patients. Clinics (Sao Paulo) 2020 Apr 17;75:e1894.

24. Kache S, Chisti MJ, Gumbo F, Mupere E, Zhi X, Nallasamy K, et al. COVID-19 PICU guidelines: for high- and limited-resource settings. Pediatr Res 2020 Nov;88(5):705-16. 